\title{
COMPACTIFICATIONS WITH ALMOST LOCALLY COMPACT OUTGROWTH
}

\author{
MARLON C. RAYBURN
}

(Communicated by Dennis Burke)

\begin{abstract}
An extension of a result of Hatzenbuhler and Mattson gives a sufficient condition that the locally compact part of an arbitrary Tichonov space has a compactification with a countably infinite outgrowth. This leads to a characterization for almost locally compact outgrowths, and some sufficient conditions for their existence. Examples are given showing the conditions are not necessary.
\end{abstract}

\section{ZIPPEN SPACES}

We shall consider compactifications to be Hausdorff. In 1935, Leo Zippen [Z] asked about Tichonov spaces with compactifications having countable outgrowths. In his honour, we refer to such objects as "Zippen" spaces. In 1966, K. D. Magill, Jr. [M] succeeded in characterizing locally compact Zippen spaces. In dealing with non-locally compact Zippen spaces, we must take account of the residue $R(X)$, the set of points at which the space $X$ is not locally compact. If $X$ is Hausdorff, $R(X)$ is always a closed subset. If $X$ is a Tichonov space and $\alpha X$ is any compactification, then $\operatorname{cl}_{\alpha X}(\alpha X \backslash X)=R(X) \cup(\alpha X \backslash X)$ [I, p. 109]. Since $\mathrm{cl}_{\alpha X}(\alpha X \backslash X)$ is a compactification of $\alpha X \backslash X$, it follows that $\mathrm{cl}_{\alpha X} R(X)=$ $R(X) \cup R(\alpha X \backslash X)$, and similarly, $\mathrm{cl}_{\alpha X} R(\alpha X \backslash X)=R(\alpha X \backslash X) \cup R^{2}(X)$, where $R^{2}(X)=R[R(X)]$ is the residue of the residue considered as a subspace of $X$. In [HM], it is shown that if $R(X)$ is a nonempty compact set, then each of the following (irreversibly) implies the next:

(i) $X$ is a Zippen space.

(ii) $X / R(X)$ is a Zippen space.

(iii) $X \backslash R(X)$ is a Zippen space.

Moreover, under the same hypotheses: $X / R(X)$ is a Zippen space if and only if $R(X)$ is a $G_{\delta}$-set in $\operatorname{cl}_{\beta X}(\beta X \backslash X)$ and the components of $R(X)$ are components of $\mathrm{cl}_{\beta X}(\beta X \backslash X)$.

Received by the editors June 6, 1988 and, in revised form, September 6, 1988.

1980 Mathematics Subject Classification (1985 Revision). Primary 54C10, 54D35, 54D40.

Key words and phrases. Compactification, countable outgrowth, almost locally compact, pseudoopen map, perfect map. 
In the process of obtaining these results, Hatzenbuhler and Mattson implicitly proved the following lemmas:

A. [HM] Let $X$ be compact Hausdorff and let $K$ be a closed $G_{\delta}$-set of $X$. Then $K$ is the union of components of $X$ if and only if there is a countable upper semicontinuous decomposition of $X$ into closed blocks having $K$ as one of the blocks.

B. [HM] Let $X$ be a Tichonov space in which $R(X)$ is a nonempty compact set. Then $\beta(X / R(X))=\beta X / R(X)$.

However, this last result, B., is just a special case of:

1. Lemma. Let $X$ be Tichonov and $K$ be any nonempty compact set in $\beta X$. Then $\beta(X \cup K / K)=\beta X / K$.

Proof. Clearly $\beta X / K$ is compact Hausdorff and contains a dense copy of $(X \cup$ $K) / K$. Hence it is a compactification of $(X \cup K) / K$. If $f \in C^{*}[(X \cup K) / K]$, let $\tau: \beta X \rightarrow \beta X / K$ be the quotient map and take $\rho$ to be the restriction of $\tau$ to $\tau^{\leftarrow}[(X \cup K) / K]$. Then $f \circ \rho \in C^{*}(X \cup K)$. Since $\beta(X \cup K)=\beta X$, we can extend $f \circ \rho$ to $\beta X$. Thus we have an extension of $f$ to $\beta X / K$, so $(X \cup K) / K$ is $C^{*}$-embedded in $\beta X / K$.

Whence their result A. yields:

2. Theorem. Let $X$ be a non-locally compact Tichonov space and let $K$ be compact in $\beta X$ so that $K \cap X=R(X)$. Then $(X \cup K) / K$ is a Zippen space if and only if $K$ is the union of connected components of $\operatorname{cl}_{\beta X}(\beta X \backslash X)$ and $\beta X \backslash(X \cup K)$ is $\sigma$-compact.

For convenience, let $X_{\beta}=X \cup \mathrm{cl}_{\beta X} R(X)$. Notice that $\beta X \backslash X_{\beta}$ is just the locally compact part of $\beta X \backslash X$.

3. Corollary. For any non-locally compact Tichonov space $X$, if $X_{\beta} / \mathrm{cl}_{\beta X} R(X)$ is a Zippen space, so is $X \backslash R(X)$. Moreover, $X_{\beta} / \mathrm{cl}_{\beta X} R(X)$ is a Zippen space if and only if $\beta X \backslash X_{\beta}$ is $\sigma$-compact and $\mathrm{cl}_{\beta X} R(X)$ is the union of components of $\mathrm{cl}_{\beta X}(\beta X \backslash X)$.

At this point, it is natural to ask when $\mathrm{cl}_{\beta X} R(X)=R\left(X_{\beta}\right)$. The answer, as shown in Theorem 8 below, involves almost locally compact outgrowths.

\section{Almost LOCALly COMPACT OUTGROWTHS}

4. Definition. If the locally compact part of $X, X \backslash R(X)$, is dense in $X$, then $X$ is an almost locally compact space.

5. Lemma. For every Hausdorff $X$, if $R(X)$ is almost locally compact, so is $X$.

Proof. Suppose there is an $X$-open $G \subseteq R(X)$. Then $G$ is $R$-open. Let $R^{2}(X)=R(R(X))$ be the residue of the residue of $X$ and suppose $G \backslash R^{2} \neq \varnothing$. Let $p \in G \backslash R^{2}$. Since $R^{2}$ is closed in $X$, there is an $R$-open $V(p)$ with $\operatorname{cl}_{R}(V)$ 
compact and contained in $G \backslash R^{2}$. Let $W$ be open in $X$ so that $W \cap R(X)=V$. Then $W \cap G$ is $X$-open and $p \in W \cap G \subseteq V$. Since $\operatorname{cl}(W \cap G) \subseteq \operatorname{cl}(V)$, compact, this contradicts $p \in R(X)$. Therefore if $G$ is open in $X$, then $G \backslash R \neq \varnothing$, whence $X$ is almost locally compact.

Let us extend our notation. Let $X$ be Tichonov and $\alpha X$ be a compactification. We define $X_{\alpha}=X \cup \mathrm{cl}_{\alpha X} R(X)$ as a subspace of $\alpha X$.

6. Theorem. For every $\alpha X, R\left(X_{\alpha}\right)$ is compact and $X_{\alpha}$ is almost locally compact.

Proof. Since $R\left(X_{\alpha}\right)$ is closed in $X_{\alpha}$ and contained in compact $\mathrm{cl}_{\alpha X} R(X)$, the result follows from 5 .

7. Lemma. Let $X$ be a non-locally compact Tichonov space and $\alpha X$ be any compactification of $X$. Then $\mathrm{cl}_{X}\left[R(X) \backslash R^{2}(X)\right] \subseteq R\left(X_{\alpha}\right)$.

Proof. We show $R \backslash R^{2} \subseteq R\left(X_{\alpha}\right)$, from whence it follows that $\mathrm{cl}_{X}\left(R \backslash R^{2}\right) \subseteq$ $\mathrm{cl}_{X_{\alpha}}\left(R \backslash R^{2}\right) \subseteq R\left(X_{\alpha}\right)$, closed in $X_{\alpha}$. Let $p \in R \backslash R^{2}$. Suppose that $p \notin R\left(X_{\alpha}\right)$, so that there is an $\left(X_{\alpha}-\right)$ open neighborhood $G$ of $p$ such that $\mathrm{cl}_{X_{\alpha}}(G)$ is compact. Since $p \notin R^{2} \cup R(\alpha X \backslash X)=\mathrm{cl}_{\alpha X} R(\alpha X \backslash X)$, we may assume $\mathrm{cl}_{X_{n}}(G) \subseteq X_{\alpha} \backslash \mathrm{cl}_{\alpha X} R(\alpha X \backslash X)=X \backslash R^{2}$, contradiction to $p \in R(X)$.

8. Theorem. Let $X$ be a non-locally compact Tichonov space. For every $\alpha$, the following are equivalent:

(a) $R^{2}(X) \subseteq R\left(X_{\alpha}\right)$.

(b) $R(X) \subseteq R\left(X_{\alpha}\right)$.

(c) $R\left(X_{\alpha}\right)=\mathrm{cl}_{\alpha X}(R(X))$.

(d) $\alpha X \backslash X$ is almost locally compact.

Proof. The equivalence of (a), (b), and (c) is immediate from the observation that $R\left(X_{\alpha}\right) \subseteq \mathrm{cl}_{\alpha X} R(X)$ is always true.

(c) $\Rightarrow$ (d) $\operatorname{cl}_{\alpha X}\left(\alpha X \backslash X_{\alpha}\right)=\left(\alpha X \backslash X_{\alpha}\right) \cup R\left(X_{\alpha}\right)=\left(\alpha X \backslash X_{\alpha}\right) \cup \mathrm{cl}_{\alpha X} R(X) \supseteq$ $\alpha X \backslash X$. But $(\alpha X \backslash X) \backslash R(\alpha X \backslash X)=\alpha X \backslash X$ is always true.

(d) $\Rightarrow$ (c) $\operatorname{cl}_{\alpha X-X}\left(\alpha X \backslash X_{\alpha}\right)=\alpha X \backslash X$, so $\mathrm{cl}_{\alpha X}\left(\alpha X \backslash X_{\alpha}\right)=\mathrm{cl}_{\alpha X}(\alpha X \backslash X)=$ $(\alpha X \backslash X) \cup R(X)=\left(\alpha X \backslash X_{\alpha}\right) \cup \mathrm{cl}_{\alpha X} R(X)$. Thus $R\left(X_{\alpha}\right)=\mathrm{cl}_{\alpha X} R(X)$.

9. Corollary. If $\alpha X \backslash X$ is almost locally compact, then $X$ is almost locally compact.

Proof. By 8, $R\left(X_{\alpha}\right)=\mathrm{cl}_{\alpha X} R(X)$. Thus $X \backslash R(X)=X_{\alpha} \backslash R\left(X_{\alpha}\right)$, so $\mathrm{cl}_{X}[X \backslash$ $R(X)]=X \cap \mathrm{cl}_{\alpha X}\left[X_{\alpha} \backslash R\left(X_{\alpha}\right)\right]=X$.

The converse of 9 is false. Every $Y$ is $\beta X \backslash X$ for almost locally compact, pseudocompact space $X=W^{*} \times \beta Y \backslash\left\{\omega_{1}\right\} \times Y$.

10. Corollary. Each of the following implies the next;

(a) $R^{2}(X)$ is almost locally compact.

(b) For every $\alpha, R(\alpha X \backslash X)$ is almost locally compact. 
(c) There exists an $\alpha$ with $R(\alpha X \backslash X)$ almost locally compact.

(d) $R(X)$ is almost locally compact.

(e) For every $\alpha, \alpha X \backslash X$ is almost locally compact.

(f) There exists an $\alpha$ with $\alpha X \backslash X$ almost locally compact.

(g) $X$ is almost locally compact.

The difference in the quantifiers in (e) and ( $f$ ) raises the question of their equivalence. As we shall see below, the implication is not reversible.

11. Theorem. Let $F_{\alpha}: \beta X \rightarrow \alpha X$ be the Čech map (fixing $X$ pointwise). If $\beta X \backslash X$ is almost locally compact, then $\alpha X / X$ is almost locally compact if and only if $f_{\alpha}\left[R\left(X_{\beta}\right)\right]=R\left(X_{\alpha}\right)$.

Proof. $f_{\alpha}\left[R\left(X_{\beta}\right)\right]=f_{\alpha}\left[\mathrm{cl}_{\beta X} R(X)\right]=\mathrm{cl}_{\alpha X} R(X)$. The result now follows from 8.

In 12 through 15, assume $X$ and $Y$ are $T_{2}$ and $f: X \rightarrow Y$ is a map (continuous surjection). Following Birke [B], let us call $f$ a $k$-map if it is a map such that the pre-image of each compact set of $Y$ is compact in $X$.

12. Theorem. If $f$ is a $k$-map, then $R(X) \subseteq f^{\leftarrow}[R(Y)]$.

Proof. Let $x \in f^{\leftarrow}[Y \backslash R(Y)]$ and $f(x)=y \in G$ (open) such that $\operatorname{cl}_{Y}(G)$ is compact. Then $x \in f^{\leftarrow}(G) \subseteq f^{\leftarrow}\left[\operatorname{cl}_{Y}(G)\right]$, compact. Therefore $x \in X \backslash R(X)$.

Recall that a map $f$ is pseudo-open if for each $y \in Y$ and $U$ open in $X$ such that $f^{\leftarrow}(y) \subseteq U$, then $y \in \operatorname{int}_{Y} f(U)$. Open maps and closed maps are pseudo-open. Since a closed $k$-map is called a perfect map, in what follows, a pseudo-open $k$-map will be called nearly perfect.

13. Theorem. If $f$ is pseudo-open and for every $y \in Y, f^{\leftarrow}(y)$ is compact, then $R(Y) \subseteq f[R(X)]$.

Proof. Suppose $y \in R(Y)$ with $f^{\leftarrow}(y) \cap R(X)=\varnothing$. For each $x \in f^{\leftarrow}(y)$, choose $X$-open $G_{x}$ with $x \in G_{x} \subseteq \operatorname{cl}\left(G_{x}\right)$, compact. Take a finite subcover of $f^{\leftarrow}(y)$ and let $G$ be its union. Then $f^{\leftarrow}(y) \subseteq G$ (open) and $\operatorname{cl}_{X}(G)$ is compact. Hence $y \in \operatorname{int}_{Y} f[G] \subseteq f\left[\mathrm{cl}_{X}(G)\right]$, contradiction to $y \in R(Y)$.

14. Corollary. If $f$ is nearly perfect, then $f[R(X)]=R(Y)$.

15. Corollary. If $f$ is an open $k$-map, then $R(X)=f^{\leftarrow}[R(Y)]$.

Proof. By 12, $R(X) \subseteq f^{\leftarrow}[R(Y)]$. Suppose $x \in X \backslash R(X)$ and $x$ has an open neighborhood $G$ with $\mathrm{cl}_{X}(G)$ compact. Then $f(x) \in f(G)$, open, and $f(G) \subseteq$ $f\left[\mathrm{cl}_{X}(G)\right]$, compact, hence $f(x) \notin R(Y)$.

16. Example. Tichonov $X$ and $Y$, and perfect $f: X \rightarrow Y$ such that $R(X)$ is a proper subset of $f^{\leftarrow}[R(Y)]$.

Let $Y=W^{*} \times \beta \mathbb{N} \backslash\left\{\omega_{1}\right\} \times \mathbb{N}$. Then $R(Y)=\left\{\omega_{1}\right\} \times(\beta \mathbb{N} \backslash \mathbb{N})$. Let $X$ be the free union of $Y$ and $\beta \mathbb{N} \backslash \mathbb{N}$. Let $f: X \rightarrow Y$ by $f \mid Y=i d_{Y}$ and 
$f(x)=\left\{\omega_{1}\right\} \times\{x\}$ for each $x \in \beta \mathbb{N} \mid \mathbb{N}$. It is easily seen that $f$ is perfect. $R(X)=R(Y)=\left\{\omega_{1}\right\} \times(\beta \mathbb{N} \backslash \mathbb{N})$, but $f^{\leftarrow}[R(Y)]$ is the free union of $R(X)$ and $\beta N \backslash N$.

Now suppose $X$ to be Tichonov. Let $f_{\alpha}: \beta X \rightarrow \alpha X$ be the Čech map and $g_{\alpha}$ be the restriction of $f_{\alpha}$ to $X_{\beta}$. Then $g_{\alpha}: X_{\beta} \rightarrow X_{\alpha}$.

17. Theorem. For any $\alpha$, if $g_{\alpha}$ is a k-map, then $\beta X \backslash X$ is almost locally compact implies $\alpha X \backslash X$ is almost locally compact.

Proof. By 12, $R\left(X_{\beta}\right) \subseteq g_{\alpha}^{\leftarrow}\left[R\left(X_{\alpha}\right)\right]$. Thus $R(X) \subseteq g_{\alpha}^{\leftarrow}\left[R\left(X_{\alpha}\right)\right]$. Since $f_{\alpha}[\beta X \backslash X]=\alpha X \backslash X$, we see $R(X) \subseteq R\left(X_{\alpha}\right)$. Therefore $\mathrm{cl}_{\alpha X} R(X)=R\left(X_{\alpha}\right)$, and $\alpha X \backslash X$ is almost locally compact.

18. Lemma. For every $\alpha$ and every $p \in X_{\alpha}, g_{\alpha}^{\leftarrow}(p)$ is compact.

19. Theorem. For any $\alpha$, if $g_{\alpha}$ is pseudo-open, then $\alpha X \backslash X$ is almost locally compact implies $\beta X \backslash X$ is almost locally compact.

Proof. Assume $\alpha X \backslash X$ to be almost locally compact. By (8) and (13), $R(X) \subseteq$ $R\left(X_{\alpha}\right) \subseteq g_{\alpha}\left[R\left(X_{\beta}\right)\right]$. The definition of $g_{\alpha}$ insures $R(X) \subseteq R\left(X_{\beta}\right)$, so an application of $(8)$ completes the proof.

20. Corollary. For each $\alpha$, if $g_{\alpha}$ is nearly perfect, then $\alpha X \backslash X$ is almost locally compact if and only if $\beta X \backslash X$ is almost locally compact.

It would be interesting to known whether $R(X)$ is almost locally compact and $X_{\beta} / R\left(X_{\beta}\right)$ is Zippen implies $X_{\alpha} / R\left(X_{\alpha}\right)$ is Zippen for all $\alpha$.

\section{EXAMPLES}

If $K$ is a compact set of $\beta X \backslash X_{\beta}$, and we let $\alpha X=\beta X / K$, then the map $g_{\alpha}: X_{\beta} \rightarrow X_{\alpha}$ is just the identity map, hence it is trivially nearly perfect. We now consider two examples where $g_{\alpha}$ is not nearly perfect.

21. Example. A space $Y$ with a compactification $\delta Y$ such that $\delta Y \backslash Y$ and $\beta Y \backslash Y$ are both almost locally compact, yet $g_{\alpha}: Y_{\beta} \rightarrow Y_{\alpha}$ is neither a pseudoopen nor a $k$-map. This shows that the sufficient hypotheses of Theorems 17 and 19 are not necessary.

Let $X \subseteq \mathbf{R}^{2}$ be given as $X=\{(x, y): 0<|x| \leq 1,|y| \leq 1\} \cup\{(0, y): y \in \mathbb{Q}$, $|y| \leq 1\} \backslash\{(x, \sin (1 / x): 0<|x| \leq 1\}$. Then $R(X)=\{(0, y): y \in \mathbb{Q},|y| \leq 1\}$. Let $\alpha X=\{(x, y):|x| \leq 1,|y| \leq 1\}$. Then $R(\alpha X \backslash X)=\{(0, y): y \in \mathbb{R} \backslash \mathbb{Q}$, $|y| \leq 1\}$. Let $p=(1 / \pi, \sin (\pi)) \in \alpha X \backslash X$, and $q=(0,1 / \sqrt{2}) \in R(\alpha X \backslash X)$. Let $\gamma X=\alpha X /\{p, q\}$ and $h_{\gamma}: \alpha X \rightarrow \gamma X$ be the quotient map. Clearly both $\alpha X \backslash X$ and $\gamma X \backslash X$ are almost locally compact.

Take $Y=W^{*} \times \alpha X \backslash\left\{\omega_{1}\right\} \times(\alpha X \backslash X)$. Then $\alpha X \backslash X=\beta Y \backslash Y$, and $\gamma X \backslash X=$ $\delta Y \backslash Y$. Let $K$ be $\operatorname{cl}_{\delta Y}\{(1 / \pi, y): 0<|y| \leq 1\}$, compact. But $g_{\alpha}^{\leftarrow}[K]$ is not compact. Again, if $G$ is open in $\delta Y$ and $f_{\delta}(p) \in G$, then there must be points of $Y$ far from $q$ contained in $f_{\delta}^{\leftarrow}[G]$. But $f_{\delta}^{\leftarrow} \circ f_{\delta}(p) \cap Y=\{q\}$, so we can 
find open $U$ in $Y_{\beta}$ with $q \in U$ and $p$ far from $U$. Thus $g_{\delta}$ cannot be pseudo-open.

The author has been unable to resolve the question as to whether the existence of an almost locally compact $\alpha X \backslash X$ by itself implies that $\beta X \backslash X$ is almost locally compact, however he conjectures it is false. The author wishes to thank R. G. Woods for the following.

22. Example. A space $X$ with a compactification $\alpha X$ such that $\sigma X \backslash X$ is nowhere locally compact, while $\beta X \backslash X$ is almost locally compact.

Let $C$ be the Cantor set. As a subspace of $[0,1], \operatorname{diam}(C)=1$. Let $\left\{A_{n}\right\}_{1}^{\infty}$ be a sequence of pairwise disjoint clopen subsets of $C$ with

(1) $0 \notin \cup_{1}^{\infty} A_{n}$.

(2) $C=\{0\} \cup \bigcup_{1}^{\infty} A_{n}$.

(3) As $n \rightarrow \infty$, diam $\left(\{0\} \cup \bigcup_{n}^{\infty} A_{k}\right) \rightarrow 0$.

Thus $\left\{\{0\} \cup \bigcup_{k=n}^{\infty} A_{k}\right\}_{n=1}^{\infty}$ is a clopen neighborhood base at 0 . Next, construct a sequence $\left\{\mathfrak{B}_{n}\right\}_{1}^{\infty}$ of partitions of $C$ into a finite family of clopen subsets of $C$ such that

(4) $\left|\mathfrak{B}_{n}\right|=2^{n}$

(5) For each $n, \mathfrak{B}_{n+1}$ refines $\mathfrak{B}_{n}$.

(6) If $\mathfrak{B}_{n}=\left\{B_{n, i}: i \leq 2^{n}\right\}$, then $\operatorname{diam}\left(B_{n, i}\right)=2^{-n}$.

Hence $C \times C=(\{0\} \times C) \cup\left[\bigcup_{n=1}^{\infty} \bigcup_{i=1}^{2^{n}} A_{n} \times B_{n, i}\right]$.

Let $Q$ be a dense, countably infinite subset of $C$. For each positive integer $n$ and each $i \leq 2^{n}$, choose $q_{n, i} \in B_{n, i} \cap Q$. Define $f: C \times C \rightarrow C$ as follows: for each $y \in C, f(0, y)=y$. If $x \neq 0$, and $(x, y) \in C \times C$, then $(x, y) \in A_{n} \times B_{n, i}$ for precisely one $n$ and one $i$. Let $f(x, y)=q_{n, i}$. Clearly $f$ is well defined.

To show: $f$ is continuous. First, if $x_{0} \neq 0$, then for any $y_{0} \in C, f$ is continuous at $\left\{x_{0}, y_{0}\right\}$. Suppose $x_{0} \in A_{m}$ and $y_{0} \in B_{m, j}$, then $f\left(x_{0}, y_{0}\right)=$ $q_{m, j}$. Suppose $E$ is clopen in $C$ and $q_{m, j} \in E$. Then $A_{m} \times B_{m, j}$ is a neighborhood of $\left(x_{0}, y_{0}\right)$ in $C \times C$ and $f\left[A_{m} \times B_{m, j}\right]=q_{m, j} \in E$. Hence $f$ is continuous at $\left(x_{0}, y_{0}\right)$.

Next, $f$ is continuous at $\left(0, y_{0}\right)$ for any $y_{0} \in C$. In this case $f\left(0, y_{0}\right)=y_{0}$. Let $E$ be a clopen neighborhood of $y_{0}$ in $C$. Since $\mathfrak{B}_{n}$ is a clopen partition of $C$, for each $n$ there will be a unique $j(n)$ between 1 and $2^{n}$ such that $y_{0} \in B_{n, j(n)}$. Let the distance from $y_{0}$ to $C \backslash E$ be $\delta(>0)$. Choose a positive integer $m$ such that $2^{-m}<\delta / 4$. Let $V=\left(\{0\} \cup \bigcup_{m}^{\infty} A_{k}\right) \times B_{m, j(m)}$, an open neighborhood of $\left(0, y_{0}\right)$ in $C \times C$. We shall show $f[V] \subseteq E$. Since $y_{0} \in$ $B_{m, j(m)}$ and $\operatorname{diam}\left(B_{m, j(m)}\right)=2^{-m}<\delta / 4$, we see that $B_{m, j(m)} \subseteq E$. Hence $f\left[\{0\} \times B_{m, j(m)}\right] \subseteq E$. Now let $(x, y) \in A_{n} \times B_{n, j(n)}$ for some $n \geq m$. Then there is some $k$ between 1 and $2^{n}$ with $y \in B_{n, k(n)}$. Since $n \geq m, \mathfrak{B}_{n}$ refines $\mathfrak{B}_{m}$ and these being partitions, it follows that $B_{n, k(n)} \subseteq B_{m, j(m)}$. Whence $f(x, y)=q_{n, k(n)} \in B_{m, j(m)} \subseteq E$. Hence $f[V] \subseteq E$, and $f$ is continuous. 
Since $f[\{0\} \times C]=C$ and $C \times C$ is compact, it follows that $f$ is a perfect map. Let $f[(C \backslash\{0\}) \times C]=Q_{0} \subseteq Q$. Since $(C \backslash\{0\}) \times C$ is dense in $C \times C$, $Q_{0}$ is dense in $C$. Since $Q_{0}$ is countable, $C \backslash Q_{0}$ is also dense in $C$. Thus $Q_{0}$ is nowhere locally compact. Let $S=f^{\leftarrow}\left[Q_{0}\right]=[(C \backslash\{0\}) \times C] \cup\left(\{0\} \times Q_{0}\right)$. Clearly $S$ is almost locally compact and $f \mid S: S \rightarrow Q_{0}$ is a perfect map.

Finally, let $X=W^{*} \times(C \times C) \backslash\left\{\omega_{1}\right\} \times S$, so $\beta X \backslash X=\left\{\omega_{1}\right\} \times S$, and $R(X)=\left\{\omega_{1}\right\} \times(C \times C \backslash S)=\left\{\omega_{1}\right\} \times\left[\{0\} \times\left(C \backslash Q_{0}\right)\right]$. By Theorem 1.3 of [R], $Q_{0}$ is homeomorphic to $\alpha X \backslash X$ for a compactification $\alpha X$ of $X$.

\section{ACKNOWLEDGMENT}

The author would like to thank the referee both for suggestions and for great patience.

\section{BIBLIOGRAPHY}

[B] D. K. Birke, Closed mappings, Surveys in general topology (G. M. Reed, editor), Academic Press, New York, 1980, pp. 1-32.

[HM] J. Hatzenbuhler and D. A. Mattson, On Hausdorff compactifications of non-locally compact spaces, Internat. J. Math. Math Sci. 2 (1979), 481-486.

[I] J. R. Isbell, Uniform spaces, Math Surveys no. 12, Amer. Math. Soc., Providence, R.I., 1964.

[M] K. D. Magill, Jr., Countable compactifications, Canad. J. Math. 18 (1966), 616-620.

[R] M. C. Rayburn, On Hausdorff compactifications, Pacific J. Math. 44 (1973), 707-714.

[Z] L. Zippen, On semicompact spaces, Amer. J. Math. 57 (1935), 327-341.

Department of Mathematics, The University of Manitoba, Winnipeg, Canada R3T 2N2 